\title{
Высокоэффективный термоэлектрический однофотонный детектор на основе гексаборидов лантана и церия
}

\author{
(C) А.С. Кузанян ${ }^{1}$, А.А. Кузанян ${ }^{1}$, В.Н. Гурин ${ }^{2, \uparrow}$, М.П. Волков ${ }^{2}$, В.Р. Никогосян ${ }^{1}$ \\ ${ }^{1}$ Институт ффизических исследований Национальной академии наук Армении, \\ 0203 Аштарак, Армения, \\ ${ }^{2}$ Физико-технический институт им. А.Ф. Иоффре Российской академии наук, \\ 194021 Санкт Петербург, Россия \\ ๑ E-mail: vladimir.gurin@mail.ioffe.ru \\ (Поступила в Редакцию 20 декабря 2018 г. \\ В окончательной редакции 24 декабря 2018 г. \\ Принята к публикации 28 декабря 2018 г.)
}

\begin{abstract}
Предлагается конструкция высокоэффективного однофотонного детектора на основе гексаборидов лантана и церия, работающего в диапазоне от инфракрасного до ультрафиолетового излучений. Приводятся данные компьютерного моделирования процессов распространения тепла в чувствительном элементе детектора после поглощения фотонов с энергией 0.5-4.13 эВ. Для достижения высокой системной эффективности детектирования фотонов в области длин волн от ближнего инфракрасного до ультрафиолетового диапазона в конструкции чувствительного элемента в качестве поглотителя и для теплоотвода предлагается использовать гексаборид лантана. Показано, что изготовленный только из гексаборидов чувствительный элемент, как однослойной, так и трехслойной конструкции, будет иметь гигагерцовую скорость счета и превышающую 90\% эффективность детектирования.
\end{abstract}

DOI: 10.21883/FTP.2019.05.47564.22

\section{1. Введение}

Разработка однофотонных детекторов электромагнитного излучения вызвана необходимостью увеличения чувствительности детекторов, используемых во многих областях науки и техники. Сегодня наиболее высокими параметрами обладают сверхпроводящие нанопроволочные детекторы [1]. Конкуренцию таким детекторам составляют термоэлектрические однофотонные детекторы (ТЭОД), которые имеют максимально простую конструкцию чувствительного элемента (см. рисунок) и отсутствие жестких требований к рабочей температуре [2]. Компьютерное моделирование процессов распределения тепла в чувствительном элементе ТЭОД с вольфрамовым (W) поглотителем показало, что подбором геометрии поглотителя и сенсора можно добиться энергетического разрешения не хуже $1 \%$ и гигагерцовой скорости счета при регистрации фотонов с энергиями 1-1000 эВ [3]. Важнейшим параметром детекторов является эффективность детектирования $(\eta)$, которая яв-

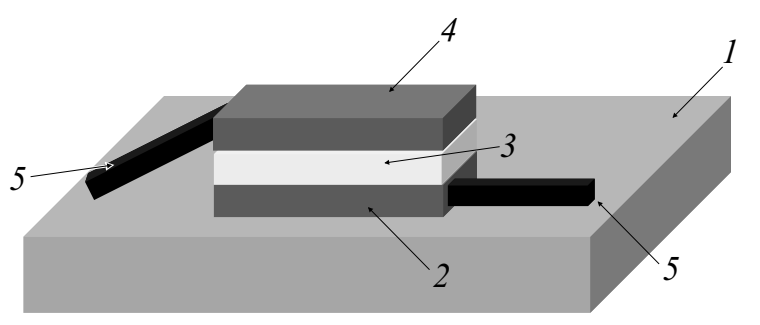

Схема трехслойного элемента чувствительного элемента ТОФД: 1 - подложка, 2 - теплоотвод, 3 - термоэлектрический сенсор, 4 - поглотитель, 5 - электрические контакты. ляется отношением числа попавших в детектор фотонов к числу им зарегистрированных. Этот параметр может быть представлен как произведение трех составляющих $\eta=\eta_{o} \cdot \eta_{a} \cdot \eta_{i}$, где $\eta_{o}$ - эффективность оптической связи фотонов и чувствительного элемента, $\eta_{a}$ - эффективность поглощения фотонов и $\eta_{i}-$ внутренняя эффективность детектирования, или вероятность регистрации уже поглощенного фотона [4]. Для длины волны 1550 нм системная эффективность сверхпроводящих однофотонных детекторов доходит до 90\% [5].

Перспективность использования гексаборидов в качестве сенсора ТЭОД рассмотрена в работе [6]. В настоящей работе методом компьютерного моделирования процессов распространения тепла исследуются характеристики ТЭОД с чувствительным элементом, состоящим только из гексаборидов $\mathrm{LaB}_{6}, \mathrm{CeB}_{6}$ и $(\mathrm{La}, \mathrm{Ce}) \mathrm{B}_{6}$ с высокой системной эффективностью детектирования в области длин волн от ближнего ИК до ультрафиолетового диапазона. Сравниваются характеристики чувствительных элементов однослойной и трехслойной конструкции.

\section{2. Выбор материала поглотителя и методика расчета}

Высокое значение $\eta_{i}$ для ТЭОД обеспечивается подбором материала термоэлектрического сенсора и выбором архитектуры чувствительного элемента. Поглотитель из $\mathrm{W}$ при соответствующем выборе толщины может обеспечить близкое к 1 значение $\eta_{a}$ для фотонов в диапазоне от ИК до жесткого рентгена, но из-за высокого коэффициента отражения $(\sim 80 \%)$ не может 
Таблица 1. Параметры использованных материалов

\begin{tabular}{|c|c|c|c|c|}
\hline \multirow{2}{*}{ Параметры } & \multicolumn{4}{|c|}{ Материал } \\
\hline & $\mathrm{LaB}_{6}$ & $(\mathrm{La}, \mathrm{Ce}) \mathrm{B}_{6}$ & $\mathrm{CeB}_{6}$ & $\mathrm{Al}_{2} \mathrm{O}_{3}$ \\
\hline Плотность, кг/м ${ }^{3}$ & 4720 & 4720 & 4800 & 4000 \\
\hline \multicolumn{5}{|c|}{$0.5 \mathrm{~K}$} \\
\hline Удельная теплоемкость, Дж/кг · К & $0.007[11]$ & $0.196[11]$ & & $9.8 \cdot 10^{-4}[13]$ \\
\hline Теплопроводность, Вт/м · К & $0.98[12]$ & $0.98[12]$ & & $40[14]$ \\
\hline Коэффициент Зеебека, мкВ/К & & $85[10]$ & & \\
\hline \multicolumn{5}{|c|}{$9 K$} \\
\hline Удельная теплоемкость, Дж/кг · K & $0.196[11]$ & & $7.3[15]$ & $0.0588[17]$ \\
\hline Теплопроводность, Вт/м · К & $100[16]$ & & $0.8[12]$ & $300[18]$ \\
\hline Коэффициент Зеебека, мкВ/К & & & $150[19]$ & \\
\hline
\end{tabular}

обеспечить высокое значение $\eta_{о}$ в ближней ИК области. Так как излучение с длиной волны 1310 и 1550 нм $(0.8$ и 0.95 эВ) используется в телекоммуникационных системах, материал поглотителя должен обладать высокой эффективностью оптической связи на данных длинах волн. Таким материалом может являться гексаборид лантана $\mathrm{LaB}_{6}$, для которого в виде монокристаллов наблюдается высокий коэффициент отражения в ближней ИК области [7], но в виде образующих покрытия наночастиц $\mathrm{LaB}_{6}$ имеет низкий коэффициент отражения $(\sim 5 \%)$ [8]. Именно такие покрытия рассмотрены нами в качестве поглотителя чувствительного элемента ТЭОД, что по сравнению с поглотителем из W может увеличить $\eta_{о}$ на длине волны 1550 нм более чем на порядок, а значения $\eta$ будут превышать $90 \%$.

Компьютерное моделирование процессов, протекающих в чувствительном элементе ТЭОД после поглощения фотонов с энергией $0.5-4.13$ эВ в центре поверхности поглотителя, проводилось на основе уравнения распространения тепла из ограниченного объема с использованием трехмерного матричного метода. Подробности примененных подходов и приближений приведены в работе [3], а использованные в расчетах физические параметры материалов - в табл. 1 .

\section{3. Результаты и их обсуждение}

Методом компьютерного моделирования исследовано поглощение фотонов с энергией $0.5-4.13$ эВ в центре поглотителя из $\mathrm{LaB}_{6}$. В случае трехслойного чувствительного элемента поглотитель, термоэлектрический слой и теплоотвод имеют площадь поверхности $10 \times 10$ мкм $^{2}$. Обозначим толщину поглотителя и теплоотвода соответственно через $Z_{1}$ и $Z_{2}$. Толщину термоэлектрического слоя в трехслойном чувствительном элементе и длину термоэлектрического мостика в однослойном чувствительном элементе обозначим через $L$. Толщина однослойного варианта чувствительного элемента была равна 0.5 мкм, площадь поверхности абсорбера $0.5 \times 5$ мкм $^{2}$. Полученные в результате расчетов характеристики чувствительного элемента имеют обозначения: $\Delta T_{m}$ - возникающая на сенсоре максимальная разность температур, $V_{m}$ - максимальное электрическое напряжение, $t_{m}$ - время достижения максимального сигнала, $t_{b}$ - время спада сигнала до фонового значения $\left(10^{-4} \mathrm{~K}\right)$ и $R=1 / t_{b}-$ скорость счета. Напряжение на сенсоре определялось из уравнения $V=\Delta T \cdot S$, где $S$ - коэффициент Зеебека. ( $\mathrm{La}, \mathrm{Ce}) \mathrm{B}_{6}$ и $\mathrm{CeB}_{6}$ имеют максимальные значения коэффициента Зеебека соответственно при 0.5 и 9 К. Именно эти температуры выбраны при расчете в качестве рабочей температуры ТЭОД.

\section{1. Чувствительный элемент ТЭОД с сенсором $(\mathrm{La}, \mathrm{Ce}) \mathrm{B}_{6}$}

Результаты компьютерного моделирования процессов распространения тепла в трехслойном и однослойном чувствительном элементе ТЭОД с термоэлектрическим сенсором $(\mathrm{La}, \mathrm{Ce}) \mathrm{B}_{6}$, поглотителем и теплоотводом $\mathrm{LaB}_{6}$ представлены в табл. 2.

По данным табл. 2 можно сделать следующие выводы. При одинаковой геометрии трехслойного чувствительного элемента параметр $t_{m}$ не зависит от энергии фотона и толщины сенсора. Расчеты 1-3 показывают, что параметры $\Delta T_{m}$ и $V_{m}$ линейно увеличиваются с увеличением энергии фотона, а скорость счета уменьшается. Фотон с большей энергией при поглощении сообщает поглотителю большее количество тепла, для ухода которого в подложку требуется больше времени. Расчеты 4-9 выполнены для фотонов с одинаковой энергией при различной толщине сенсора. Можно заметить, что с уменьшением $L$ параметры $\Delta T_{m}, V_{m}$ и $t_{b}$ уменьшаются, а скорость счета увеличивается.

Расчеты 10-14 для однослойного чувствительного элемента показывают, что параметры $\Delta T_{m}, t_{m}$ и $V_{m}$ слабо зависят от длины термоэлектрического сенсора. 
Таблица 2. Характеристики чувствительного элемента ТЭОД с сенсором (La,Ce)B 6 при $T=0.5 \mathrm{~K}$

\begin{tabular}{|c|c|c|c|c|c|c|c|}
\hline № & $L$, мкм & $E$, эВ & $\Delta T_{m}, \mathrm{MK}$ & $t_{m}$, пс & $V_{m}$, мкВ & $t_{b}$, пс & $R, \Gamma \Gamma ц$ \\
\hline \multicolumn{8}{|c|}{ Трехслойный чувствительный элемент, $Z_{1}=0.5$ мкм, $Z_{2}=1$ мкм } \\
\hline 1 & 1 & 4.13 & 53.74 & 1.5 & 4.568 & 1950 & 0.51 \\
\hline 2 & 1 & 0.8 & 10.4 & 1.5 & 0.884 & 201.9 & 4.95 \\
\hline 3 & 1 & 0.5 & 6.5 & 1.5 & 0.553 & 136.5 & 7.3 \\
\hline 4 & 4 & 0.8 & 10.4 & 1.5 & 0.884 & 304.5 & 3.28 \\
\hline 5 & 2 & 0.8 & 10.4 & 1.5 & 0.884 & 283.8 & 3.5 \\
\hline 6 & 0.6 & 0.8 & 10.03 & 1.5 & 0.853 & 165.3 & 6 \\
\hline 7 & 0.4 & 0.8 & 9.51 & 1.5 & 0.808 & 125.7 & 8 \\
\hline 8 & 0.2 & 0.8 & 8.12 & 1.5 & 0.69 & 58.5 & 17.1 \\
\hline 9 & 0.1 & 0.8 & 5.97 & 1.5 & 0.508 & 19.5 & 51.3 \\
\hline \multicolumn{8}{|c|}{ Однослойный чувствительный элемент } \\
\hline 10 & 4 & 0.8 & 52.2 & 0.66 & 4.437 & 84.6 & 11.82 \\
\hline 11 & 2 & 0.8 & 52.2 & 0.66 & 4.437 & 84.6 & 11.82 \\
\hline 12 & 1 & 0.8 & 52.2 & 0.654 & 4.437 & 73.71 & 13.57 \\
\hline 13 & 0.5 & 0.8 & 52.2 & 0.654 & 4.437 & 37.68 & 25.54 \\
\hline 14 & 0.1 & 0.8 & 52.17 & 0.651 & 4.434 & 14.52 & 68.87 \\
\hline
\end{tabular}

Таблица 3. Характеристики чувствительного элемента ТЭОД с сенсором $\mathrm{CeB}_{6}$ при $T=9 \mathrm{~K}$

\begin{tabular}{|c|c|c|c|c|c|c|c|}
\hline № & $L$, мкм & $E$, эВ & $\Delta T_{m}, \mathrm{MK}$ & $t_{m}, \Pi \mathrm{c}$ & $V_{m}$, мкВ & $t_{b}, \Pi \mathrm{c}$ & $R, \Gamma \Gamma ц$ \\
\hline \multicolumn{8}{|c|}{ Трехслойный чувствительный элемент, $Z_{1}=0.5$ мкм, $Z_{2}=1$ мкм } \\
\hline 15 & 1 & 4.13 & 4.82 & 279 & 0.723 & 10000 & 0.1 \\
\hline 16 & 1 & 0.8 & 0.93 & 279 & 0.14 & 4416 & 0.23 \\
\hline 17 & 1 & 0.5 & 0.58 & 279 & 0.087 & 2800 & 0.36 \\
\hline 18 & 0.1 & 4.13 & 1.12 & 200.8 & 0.168 & 1850 & 0.54 \\
\hline 19 & 0.1 & 0.8 & 0.2 & 200.8 & 0.029 & 468 & 2.14 \\
\hline 20 & 0.1 & 0.5 & 0.14 & 200.8 & 0.02 & 454.56 & 2.2 \\
\hline 21 & 0.6 & 0.8 & 0.93 & 279 & 0.14 & 3100 & 0.32 \\
\hline 22 & 0.4 & 0.8 & 0.26 & 264 & 0.039 & 1300 & 0.77 \\
\hline 23 & 0.2 & 0.8 & 0.25 & 231 & 0.038 & 831 & 1.2 \\
\hline \multicolumn{8}{|c|}{ Однослойный чувствительный элемент } \\
\hline 24 & 1 & 0.8 & 1.61 & 164.4 & 0.242 & 3157.2 & 0.32 \\
\hline 25 & 0.5 & 0.8 & 1.61 & 164.4 & 0.242 & 2578.2 & 0.39 \\
\hline 26 & 0.1 & 0.8 & 1.57 & 153.3 & 0.235 & 1521.6 & 0.65 \\
\hline 27 & 0.05 & 0.8 & 1.5 & 146.1 & 0.225 & 1513.2 & 0.66 \\
\hline
\end{tabular}

Однако с уменьшением $L$ параметр $t_{b}$ уменьшается, а $R$ увеличивается. При $L=0.1$ мкм достигается скорость счета 68.87 ГГц, что несколько больше чем в случае трехслойного чувствительного элемента. Отметим, что при выбранных размерах чувствительного элемента и одинаковой энергии фотона у однослойного чувствительного элемента параметры $\Delta T_{m}$ и $V_{m}$ почти на порядок больше.

\section{2. Чувствительный элемент ТЭОД с сенсором $\mathrm{CeB}_{6}$}

Результаты компьютерного моделирования процессов распространения тепла в трехслойном и однослойном чувствительном элементе ТЭОД с поглотителем и теплоотводом $\mathrm{LaB}_{6}$ и термоэлектрическим сенсором $\mathrm{CeB}_{6}$ представлены в табл. 3. Расчеты 15-17 и 18-20 выполнены соответственно для толщин термоэлектрика 1 и 0.1 мкм. Для сенсора $\mathrm{CeB}_{6}$ параметры $\Delta T_{m}$ и $V_{m}$ также линейно увеличиваются с увеличением энергии фотона, а скорость счета уменьшается. Параметр $t_{m}$ при прочих равных условиях не зависит от энергии фотона. Результаты расчетов при одинаковой энергии фотона $E=0.8$ эВ показывают, что с уменьшением $L$ параметры $\Delta T_{m}, V_{m}, t_{m}$ и $t_{b}$ уменьшаются, а $R$ увеличивается, достигая при $L=0.1$ мкм значения 2.14ГГц. Такие же закономерности наблюдаются и для однослойного чувствительного элемента. По расчетам 24-27 видно, что однослойный чувствительный элемент по сравнению с 
трехслойным обеспечивает сигнал более высокого уровня, но меньшую скорость счета. Очевидно, что величина параметров времени спада сигнала до фонового значения и скорости счета может изменяться в несколько раз в зависимости от выбранного при моделировании уровня фона. Значение $10^{-4} \mathrm{~K}$ выбрано нами исходя из того, что на данном уровне можно обеспечить на сенсоре детектора однородность и постоянство температуры. В эксперименте быстродействие реального детектора будет зависеть также от быстродействия системы регистрации сигнала.

\section{4. Заключение}

Рассчитаны характеристики чувствительного элемента ТЭОД с поглотителем $\mathrm{LaB}_{6}$ и сенсором $(\mathrm{La}, \mathrm{Ce}) \mathrm{B}_{6}$ и $\mathrm{CeB}_{6}$. Полученные результаты показывают, что такой детектор может регистрировать одиночные фотоны в области длин волн от ближнего ИК до УФ для обоих типов сенсоров. При этом обеспечивается гигагерцовая скорость счета и системная эффективность детектирования более $90 \%$.

Сравнение полученных в результате компьютерного моделирования параметров для сенсоров $(\mathrm{La}, \mathrm{Ce}) \mathrm{B}_{6}$ и $\mathrm{CeB}_{6}$ показывает, что $(\mathrm{La}, \mathrm{Ce}) \mathrm{B}_{6}$ обеспечивает получение более высокого сигнала и большей скорости счета. Преимуществом $\mathrm{CeB}_{6}$ сенсора является более высокая рабочая температура. Таким образом, как однослойная, так и трехслойная конструкция чувствительного элемента ТЭОД с термоэлектрическими сенсорами $(\mathrm{La}, \mathrm{Ce}) \mathrm{B}_{6}$ и $\mathrm{CeB}_{6}$ обладают характеристиками, позволяющими изготовить на их основе однофотонные детекторы электромагнитного излучения с большой скоростью счета.

Исследование выполнено при финансовой поддержке Государственного комитета по науке МОН РА в рамках научного проекта № 18T-2F134.

\section{Список литературы}

[1] L. You. Supercond. Sci. Technol., 31, 040503 (2018).

[2] A. Gulian, K. Wood, D. van Vechten, G. Fritzdet. J. Mod. Opt., 51, 1467 (2004).

[3] A. Kuzanyan, V. Nikoghosyan, A. Kuzanyan. Sensors and Transducers, 191, 57 (2015).

[4] F. Marsili, V.B. Verma, J.A. Stern, S. Harrington, A.E. Lita, T. Gerrits, I. Vayshenker, B. Baek, M.D. Shaw, R.P. Mirin, S.W. Nam. Nature Photonics, 7, 210 (2013).

[5] W.J. Zhang, L.X. You, H. Li, J. Huang, C.L. Lv, L. Zhang, X.Y. Liu, J.J. Wu, Z. Wang, X.M. Xie. Sci. China-Phys. Mech. Astron., 60, 120314 (2017).

[6] А.С. Кузанян, А.А. Кузанян, В.Р. Никогосян, В.Н. Гурин, М.П. Волков. ФТП, 51 (7), 908 (2017).

[7] В.Н. Гурин, М.М. Корсукова, М.Г. Карин, К.К. Сидорин, И.А. Смирнов, А.И. Шелых. ФТТ, 22, 715 (1980).

[8] H. Takeda, H. Kuno, K. Adachi. J. Am. Ceram. Soc., 91, 2897 (2008).

[9] M.M. Korsukova, V.N. Gurin, Sh. Otani, Y. Ishizava. Sol. St. Commun., 99, 215 (1996).
[10] K. Winzer. Sol. St. Commun., 16, 521 (1975).

[11] М.А. Анисимов, В.В. Глушков, А.В. Богач, С.В. Демишев, Н.А. Самарин, С.Ю. Гаврилкин, К.В. Мицень, Н.Ю. Шицевалова, А.В. Левченко, В.Б. Филиппов, С. Габони, К. Флахбарт, Н.Е. Случанко. ЖЭТФ, 143, 877 (2013).

[12] Y. Peysson, C. Ayache, B. Salce. J. Magn. Magn. Mater., 59, 33 (1986).

[13] G.T. Furukawar, T.B. Douglas, R.E. McCoske Yr, D.C. Ginnings. J. Research National Bureau Stand., 57, 67 (1956).

[14] http://www.phys.ufl.edu/ireu/IREU2013/pdf_reports/Allen_ Scheie_FinalReport.pdf

[15] T. Fujita, M. Suzuki, T. Komatsubara, S. Kunii, T. Kasuya, T. Ohtsuka. Sol. St. Commun., 35, 569 (1980).

[16] П.А. Попов, В.В. Новиков, А.А. Сидоров, Е.В. Максименко. Неорг. матер., 43, 1324 (2007).

[17] D.A. Ditmars, S. Ishihara, S.S. Chang, G. Bernstein, E.D. West. J. Research National Bureau Stand., 87, 159 (1982).

[18] R. Berman, E.L. Foster, J.M. Ziman. Ser. A, 231, 130 (1955).

[19] V. Petrosyan, V. Vardanyan, V. Kuzanyan, M. Konovalov, V. Gurin, A. Kuzanyan. Sol. St. Sci., 14, 1653 (2012).

Редактор А.Н. Смирнов

\section{High efficiency thermoelectric single-photon detector on the base of lanthanum and cerium hexaborides}

\author{
A.S. Kuzanyan ${ }^{1}$, A.A. Kuzanyan ${ }^{1}$, V.N. Gurin ${ }^{2}$, \\ M.P. Volkov ${ }^{2}$, V.R. Nikoghosyan ${ }^{1}$ \\ ${ }^{1}$ Institute for Physical Research, \\ 0203 Ashtarak-2, Republic of Armenia \\ 2 loffe Institute, \\ 194021 St. Petersburg, Russian Federation
}

\begin{abstract}
The results of computer simulation of heat distribution processes in sensitive element of a thermoelectric single-photon detector after absorption of photons with energy of $0.5-4.13 \mathrm{eV}$ are presented. To achieve high system efficiency of photon detection in the wavelength range from the near infrared to the ultraviolet, it is proposed to use lanthanum hexaboride as an absorber and heat sink in the design of the sensitive element. It is shown that a sensitive element made only from hexaborides of both single-layer and three-layer construction will have a gigahertz count rate and a detection efficiency exceeding $90 \%$.
\end{abstract}

